\title{
Dispensing Patterns of Ranibizumab and Aflibercept for the Treatment of Neovascular Age-Related Macular Degeneration: A Retrospective Cohort Study in Australia
}

\author{
Adrian Skelly (D) Hans-Joachim Carius · Vladimir Bezlyak •
}

Fred K. Chen

Received: May 3, 2017 / Published online: November 21, 2017

(c) The Author(s) 2017. This article is an open access publication

\section{ABSTRACT}

Introduction: Anti-vascular endothelial growth factor therapy is the standard of care for neovascular age-related macular degeneration (nAMD). The dosage of two licensed agents, ranibizumab and aflibercept, was established through clinical trials; however, it is unclear if either agent is administered as recommended in routine clinical practice. Using pharmacy claims data, we investigated if the dispensing patterns of ranibizumab differ from those of aflibercept 6 and 12 months after treatment initiation.

Methods: Prescription data retrieved from the Australian IMS ${ }^{\circledR}$ AUS LRx database were used to identify nAMD patients with one or more claims for ranibizumab or aflibercept between

Enhanced content To view enhanced content for this article go to http://www.medengine.com/Redeem/ 01BCF0607CD3A1F5.

A. Skelly $(\bowtie) \cdot$ V. Bezlyak

Novartis Pharma AG, Basel, Switzerland

e-mail: adrian.skelly@novartis.com

H.-J. Carius

QuintilesIMS, Frankfurt, Germany

F. K. Chen

Centre for Ophthalmology and Visual Science (Incorporating Lions Eye Institute), The University

of Western Australia, Perth, WA, Australia

F. K. Chen

Department of Ophthalmology, Royal Perth

Hospital, Perth, WA, Australia
December 1, 2012, and March 31, 2015, with follow-up of at least 6 months. The number of ranibizumab and aflibercept units dispensed was adjusted for baseline patient Medication-Based Disease Burden Index (MBDBI) scores. No difference in the number of ranibizumab versus aflibercept units dispensed was concluded if the 95\% confidence interval (CI) limits of the adjusted mean difference between the study cohorts were 1.00 unit or less.

Results: Baseline patient MBDBI scores were similar for the ranibizumab $(N=1235)$ and aflibercept $(N=959)$ cohorts. The adjusted mean (standard deviation) number of units dispensed was 5.3 (1.3) versus 5.1 (1.4) at month 6 and 8.9 (2.2) versus 8.9 (2.3) at month 12. The $95 \%$ CI limits of the adjusted mean difference did not exceed 1.00 unit dispensed at either time point: $95 \%$ CI of 0.09 to 0.32 for an adjusted mean difference of 0.20 at month 6 and -0.23 to 0.30 for an adjusted mean difference of 0.04 at month 12. Mean (standard deviation) dispensing intervals were comparable for both cohorts: 35.3 (19.2) days versus 36.8 (20.0) days at month 6 (adjusted mean difference -1.59 days; $95 \%$ CI -2.51 to -0.67 days) and 41.2 (20.9) days versus 41.6 (20.4) days at month 12 (adjusted mean difference -0.40 days; $95 \%$ CI -1.70 to 0.91 days).

Conclusions: Ranibizumab and aflibercept are dispensed in a similar manner by Australian pharmacies during the first year of treatment. Funding: Novartis Pharma AG. 
Keywords: Aflibercept; Anti-vascular endothelial growth factor; Dispensing pattern; Neovascular age-related macular degeneration; Ophthalmology; Ranibizumab; Vascular endothelial growth factor

\section{INTRODUCTION}

Age-related macular degeneration (AMD), a chronic disorder of the retina, is a leading cause of progressive vision loss in individuals aged 65 years or older in the industrialized world, including Australia [1, 2]. In 2010, it was estimated that more than one million Australians had AMD, resulting in annual economic losses of more than $\$ 5$ billion [3]. There are two main types of AMD: "dry" AMD, or atrophic AMD, and "wet" AMD, or neovascular AMD (nAMD). Of these, nAMD represents $10-20 \%$ of all AMD cases yet is responsible for $80-90 \%$ of the severe vision loss associated with AMD [4]. nAMD is characterized by the formation of abnormal blood vessels from the choroid toward the retina (a process called "choroidal neovascularization"), which can exude fluid, causing progressive degeneration of the photoreceptors and the retinal pigment epithelium. Without early diagnosis and treatment, nAMD can progress rapidly, leading to severe visual impairment and irreversible blindness in the affected eye [5].

The introduction of anti-vascular endothelial growth factor (VEGF) therapy has revolutionized the treatment of nAMD, contributing to the visual improvement of patients while reducing public health expenditure due to the consequences of vision impairment $[3,6]$. In Australia, the standard care for the treatment of nAMD involves intravitreal injections of licensed anti-VEGF agents, ranibizumab or aflibercept. Ranibizumab was first listed on the Australian Pharmaceutical Benefits Scheme (PBS) in August 2007, with aflibercept first listed in December 2012 [7]. Despite the recommended 4-weekly (i.e., monthly) dosing interval on the product label, a "treat-and-extend" (T\&E) protocol (approved in 2016) is the predominant treatment regimen used for ranibizumab in Australian clinics-after three consecutive monthly injections during the loading phase, retreatment intervals may be extended by $1-2$ weeks in a stepwise manner (capped at 12 weeks) at the treating physician's discretion using retreatment criteria based on morphological and visual parameters without the need for additional assessment visits $[8,9]$. For aflibercept, the recommended dosing regimen on the product label is three monthly loading doses followed by injection at fixed intervals of 8 weeks [10]. Accordingly, clinicians who follow the recommended dosing as per the product label would be expected to administer 13 ranibizumab or 8 aflibercept doses for each affected eye in the first year of treatment; in contrast, T\&E regimens require a minimum of seven injections of both agents during the same period [11, 12].

Identifying the optimum treatment regimen for $n A M D$ patients remains an ongoing challenge, the primary goal of which is to minimize the number of intravitreal injections and patient visits without compromising visual improvement. Available clinical trial evidence suggests that the recommended dosing frequency for aflibercept is less burdensome than that for ranibizumab $[12,13]$; however, analysis of observational data retrieved from the Fight Retinal Blindness registry demonstrated that visual outcomes and the number of injections did not differ between ranibizumab- and aflibercept-treated patients 12 months after the initiation of treatment [14]. Recent studies have also explored the use of physician-level medical claims databases and electronic medical records (EMR) to assess the real-world use of anti-VEGF agents for the treatment of nAMD and other retinal diseases [14-20]. Several of these studies have shown that, on average, the number of ranibizumab injections received by nAMD patients in the first year of treatment was lower than that expected if patients had been treated strictly according to the label instructions [14-16].

To better understand the real-world use of ranibizumab and aflibercept for the treatment of nAMD, we assessed the dispensing patterns of both agents 6 and 12 months after the index date using prescription data provided by Australian pharmacies, where the index date is defined as the date of the first recorded dispensing of 
ranibizumab or aflibercept to a patient by a pharmacy. The primary objective of this study was to analyze and compare dispensing patterns of ranibizumab and aflibercept on the basis of the mean number of units dispensed at these post-index assessment time points. We concluded that the number of dispensed units of ranibizumab was not greater than the number of dispensed units of aflibercept if the 95\% confidence interval (CI) limits of the cohort adjusted mean differences (ranibizumab-aflibercept) did not exceed 1.00 unit.

\section{METHODS}

\section{Ethics Statement}

This study was designed, conducted, and reported in accordance with the guidelines for good pharmacoepidemiology practices of the International Society for Pharmacoepidemiology [21], the Strengthening the Reporting of Observational Studies in Epidemiology guidelines [22], and the ethical principles stated in the Declaration of Helsinki [23]. For this study, all patient identifiers were removed; thus, formal patient informed consent was not required.

\section{Study Design and Patient Selection}

This retrospective cohort study used prescription data extracted from the IMS ${ }^{\circledR}$ AUS LRx $09 / 2015$ database managed and provided by QuintilesIMS (http://www.imshealth.com). The study included patients with a pharmacy record of receiving at least one unit of ranibizumab (PBS code $10138 \mathrm{~N}$ or 1382R) or aflibercept (PBS code $2168 \mathrm{D}$ ) in the database during the identification period of December 1, 2012 to March 31,2015 , inclusive. The costs for both antiVEGF agents were reimbursed by the PBS for the treatment of nAMD only during this period [24-27]. Patients were required to not have any recorded anti-VEGF therapy in the 6 months before the index date. Inclusion criteria also required that patients received their index agent (i.e., the recorded anti-VEGF agent dispensed to a patient on the index date) from a pharmacy that submitted at least one claim of any pharmaceutical agent to the database each calendar month for a period of six consecutive months ( 1 month equals 30 days) before and after the index date-a total of 13 months including the month with the claim indicating the index date. This criterion ensured that the pharmacy was actively submitting claims during the entire pre- and post-index period. Patients were excluded from the study if they had: (1) a record of receiving both ranibizumab and aflibercept on the index date; (2) a record of receiving any anti-VEGF therapy before December 1, 2012; or (3) a record of receiving bevacizumab during the 6 months before the index date, including the index date, and any time during the follow-up period.

The data were divided into ranibizumab and aflibercept cohorts according to the recorded index agent received by a patient. Following application of the inclusion and exclusion criteria, the final study cohorts-that is, those patients who persisted with their respective index agent for 6 and 12 months after the index date-were identified.

\section{Study Objectives and Variables}

Patient characteristics such as age and sex were not well populated in the IMS $^{\circledR}$ AUS LRx 09/2015 database, and were therefore not computed at the index date. Instead, the Medication-Based Disease Burden Index (MBDBI) [28] was used as a metric to compare baseline characteristics of the ranibizumab and aflibercept cohorts. The MBDBI includes drugs and drug classes corresponding to 20 chronic disease conditions and their associated weightings. If a patient's medication regimen contained any MBDBI-listed disease, that patient was assigned a disutility weighting corresponding to a given disease condition. The total score on the MBDBI was calculated as the sum of weightings for the various drugs and drug classes present in a given regimen. The MBDBI score was calculated on the basis of all medications co-prescribed in the 6 months before and including the index date. 
The primary study objective was to demonstrate that the number of dispensed units of ranibizumab was not greater than the number of dispensed units of aflibercept. For this, we compared the mean number of units of each agent dispensed (i.e., the primary study outcome) for the final study cohorts at 6 and 12 months after the index date (herein referred to as month 6 and month 12). We concluded that there was no difference in the number of dispensed units of ranibizumab versus aflibercept if the 95\% CI limits of the adjusted mean difference (ranibizumab-aflibercept) did not exceed 1.00 unit, which was deemed to be meaningful from a pharmacy administration and health authority reimbursement scheme perspective [29].

The secondary outcomes of the study included the dispensing interval (i.e., number of days between recorded receipts of ranibizumab or aflibercept) for the final study cohorts at month 6 and month 12. Additional secondary outcomes were the proportion of patients who: (1) persisted with the index agent; (2) switched the index agent to the comparator agent; (3) discontinued use of the index agent (defined as a patient having no record of receiving units of the index agent for at least 120 days, i.e., 4 months post-index date); and (4) restarted use of the index agent during the 6- and 12-month post-index periods. These groups were mutually exclusive; for example, patients who were recorded as switching from or restarting use of their index agent were not considered as having discontinued use of their index agent in the analysis presented here.

\section{Statistical Analysis}

All statistical tests were performed with SAS version 9.4 (SAS Institute, Cary, NC, USA), with, where necessary, the ranibizumab cohort as a reference. Descriptive data are reported as the mean and standard deviation (SD) or proportions (percentages). Difference in proportion tests were used to compare the proportion of patients displaying comorbidities in the MBDBI for each anti-VEGF cohort. Analysis of variance was used to assess the difference in the number of dispensed units between the two final study cohorts. Estimation of the mean difference in days between dispensed units for the ranibizumab and aflibercept cohorts was performed with generalized estimating equations. All adjusted models controlled for baseline MBDBI scores. Kaplan-Meier curves and log-rank tests were used to calculate the time to discontinuation of use of or switching from the index agent at month 6 and month 12 . The risk of discontinuation of use of index agent or switching from the index agent to the comparator agent at both assessment time points was calculated with Cox proportional hazards models.

\section{RESULTS}

\section{Study Population and Cohorts}

Among the patients registered in the IMS ${ }^{\circledR}$ AUS LRx 09/2015 database, 5130 patients had at least one record of receiving ranibizumab and 2639 patients had at least one record of receiving aflibercept in the identification period. The primary analysis set comprised those patients with no recorded anti-VEGF therapy 6 months before the index date and who had received their index agent from a dispensing pharmacy that had submitted at least one claim (for any pharmaceutical agent) to the database each calendar month for 6 and 12 consecutive months after the index date. For ranibizumab, the respective patient numbers were 2412 (47.0\%) and 1758 (34.3\%); for aflibercept, the corresponding patient numbers were 1711 (64.8\%) and 1119 (42.4\%). Application of the additional inclusion and exclusion criteria yielded final month 6 study cohorts comprising 1235 ranibizumab patients (24.1\%) and 959 aflibercept patients (36.3\%); the final month 12 study cohorts comprised 578 ranibizumab patients (11.3\%) and 483 aflibercept patients $(18.3 \%)$. The primary outcome of the study together with the mean dispensing interval was measured and compared using these final study cohorts (Fig. 1). 


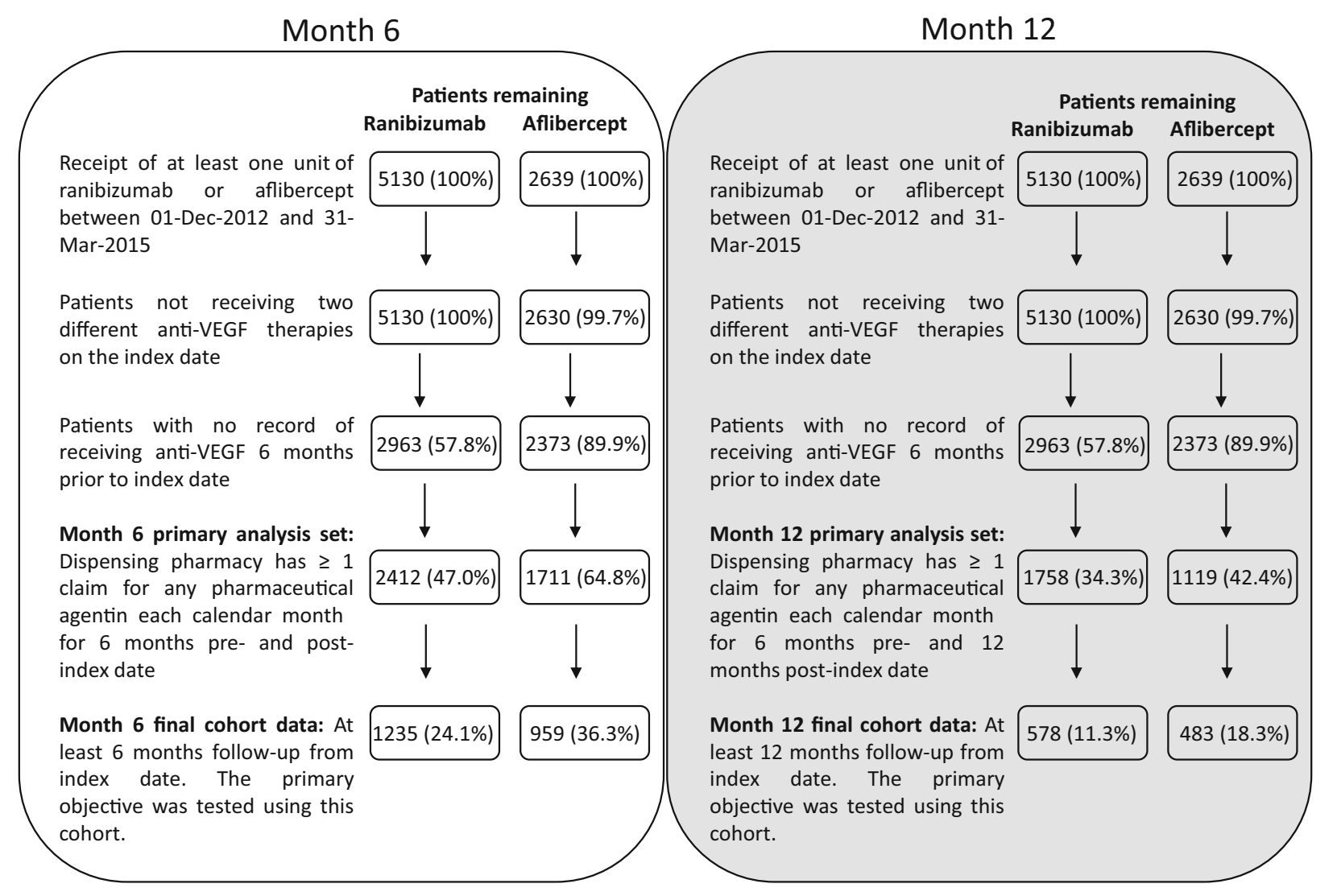

Fig. 1 Development and attrition of the ranibizumab and aflibercept cohorts at month 6 and month 12. VEGF vascular endothelial growth factor

\section{Primary Study Outcome}

Baseline patient comorbidities as determined by the MBDBI were similar for the final ranibizumab and aflibercept cohorts at both assessment time points (Table 1). The mean (SD) number of units dispensed for the ranibizumab and aflibercept cohorts at month 6 was 5.3 (1.3) and 5.1 (1.4), respectively (adjusted mean difference 0.2 ; $95 \%$ CI 0.09 to 0.32). At month 12, the values were 8.9 (2.2) for the ranibizumab cohort and 8.9 (2.3) for the aflibercept cohort (adjusted mean difference $0.04 ; 95 \% \mathrm{CI}-0.23$ to 0.30 ) (Table 2, Fig. 2).

The frequency distributions for the number of units dispensed at month 12 for the ranibizumab and aflibercept cohorts were comparable (Fig. 3). Most ranibizumab patients (93.9\%, $543 / 578$ ) were treated less frequently than the label-recommended monthly dosing schedule, while half of the aflibercept patients $(50.1 \%$, 242/483) required more frequent treatment than the label recommendation.

\section{Secondary Study Outcomes}

The mean (SD) dispensing interval for the ranibizumab cohort was similar to that for the aflibercept cohort at both assessment time points: 35.3 (19.2) days versus 36.8 (20.0) days (adjusted mean difference of -1.59 days; $95 \%$ CI -2.51 to -0.67 days) at month 6 and 41.2 (20.9) days versus 41.6 (20.4) days (adjusted mean difference of -0.40 days; $95 \% \mathrm{CI}-1.70$ to 0.91 days) at month 12 (Table 3, Fig. 4).

Comparison of the proportion of patients who persisted with, switched from, discontinued use of, or restarted use of the index agent 
Table 1 Number and proportion of patients displaying comorbidities of the Medication-Based Disease Burden Index

\begin{tabular}{|c|c|c|c|}
\hline Comorbidities & Ranibizumab cohort $N(\%)$ & Aflibercept cohort $N(\%)$ & $P$ \\
\hline Month 6 (180 days) & $N=1235$ & $N=959$ & \\
\hline No disease & $1011(81.86 \%)$ & $809(84.36 \%)$ & 0.12 \\
\hline Alzheimer's disease and other dementias & $8(0.65 \%)$ & $3(0.31 \%)$ & 0.37 \\
\hline Asthma & $69(5.59 \%)$ & $52(5.42 \%)$ & 0.87 \\
\hline Benign prostatic hypertrophy & $10(0.81 \%)$ & $7(0.73 \%)$ & 0.83 \\
\hline Cerebrovascular disease & $5(0.40 \%)$ & $2(0.21 \%)$ & 0.48 \\
\hline Cirrhosis & $0(0.00 \%)$ & $0(0.00 \%)$ & \\
\hline COPD & $7(0.57 \%)$ & $6(0.63 \%)$ & 0.86 \\
\hline Diabetes mellitus & $66(5.34 \%)$ & $41(4.28 \%)$ & 0.25 \\
\hline Epilepsy & $7(0.57 \%)$ & $6(0.63 \%)$ & 0.86 \\
\hline Hepatitis B & $0(0.00 \%)$ & $0(0.00 \%)$ & \\
\hline Hepatitis C & $0(0.00 \%)$ & $0(0.00 \%)$ & \\
\hline HIV infection & $0(0.00 \%)$ & $0(0.00 \%)$ & \\
\hline Hypertensive heart disease & $3(0.24 \%)$ & $3(0.31 \%)$ & 1.00 \\
\hline Ischemic heart disease & $17(1.38 \%)$ & $9(0.94 \%)$ & 0.35 \\
\hline Malignant neoplasms & $18(1.46 \%)$ & $6(0.63 \%)$ & 0.06 \\
\hline Nephritis/nephrosis & $0(0.00 \%)$ & $0(0.00 \%)$ & \\
\hline Parkinson's disease & $6(0.49 \%)$ & $2(0.21 \%)$ & 0.48 \\
\hline Peptic ulcer disease & $2(0.16 \%)$ & $0(0.00 \%)$ & 0.51 \\
\hline Rheumatic heart disease & $1(0.08 \%)$ & $3(0.31 \%)$ & 0.32 \\
\hline Skin diseases & $79(6.40 \%)$ & $50(5.21 \%)$ & 0.24 \\
\hline Tuberculosis & $0(0.00 \%)$ & $0(0.00 \%)$ & \\
\hline Month 12 (360 days) & $N=578$ & $N=483$ & \\
\hline No disease & $475(82.18 \%)$ & $406(84.06 \%)$ & 0.42 \\
\hline Alzheimer's disease and other dementias & $7(1.21 \%)$ & $2(0.41 \%)$ & 0.19 \\
\hline Asthma & $33(5.71 \%)$ & $25(5.18 \%)$ & 0.70 \\
\hline Benign prostatic hypertrophy & $2(0.35 \%)$ & $2(0.41 \%)$ & 1.00 \\
\hline Cerebrovascular disease & $2(0.35 \%)$ & $1(0.21 \%)$ & 1.00 \\
\hline Cirrhosis & $0(0.00 \%)$ & $0(0.00 \%)$ & \\
\hline COPD & $5(0.87 \%)$ & $3(0.62 \%)$ & 0.73 \\
\hline Diabetes mellitus & $27(4.67 \%)$ & $19(3.93 \%)$ & 0.56 \\
\hline
\end{tabular}


Table 1 continued

\begin{tabular}{lccc}
\hline Comorbidities & Ranibizumab cohort $\boldsymbol{N}(\%)$ & Aflibercept cohort $\boldsymbol{N}(\%)$ & $\boldsymbol{P}$ \\
\hline Epilepsy & $3(0.52 \%)$ & $0(0.00 \%)$ & 1.00 \\
Hepatitis B & $0(0.00 \%)$ & $0(0.00 \%)$ & $0(0.00 \%)$ \\
Hepatitis C & $0(0.00 \%)$ & $3(0.62 \%)$ & $5(1.04 \%)$ \\
HIV infection & $0(0.00 \%)$ & $3(0.62 \%)$ & 0.66 \\
Hypertensive heart disease & $2(0.35 \%)$ & $0(0.00 \%)$ & 0.25 \\
Ischemic heart disease & $11(1.90 \%)$ & $1(0.21 \%)$ & 0.36 \\
Malignant neoplasms & $7(1.21 \%)$ & $0(0.00 \%)$ & 1.00 \\
Nephritis/nephrosis & $0(0.00 \%)$ & $2(0.41 \%)$ \\
Parkinson's disease & $1(0.17 \%)$ & $28(5.80 \%)$ \\
Peptic ulcer disease & $0(0.00 \%)$ & $0(0.00 \%)$ \\
Rheumatic heart disease & $1(0.17 \%)$ & $3(6.06 \%)$ & 0.59 \\
Skin diseases & $0(0.00 \%)$ & 0.86 \\
Tuberculosis & &
\end{tabular}

Differences in proportion tests were used to compare the proportion of patients displaying the different comorbidities for each treatment cohort. Analyses were conducted with the final month 6 and month 12 study cohorts

was performed on the primary analysis set. The proportions of patients in the corresponding categories for the ranibizumab and aflibercept cohorts were largely comparable at both assessment time points, with some exceptions (Table 4). Firstly, a lower proportion of ranibizumab patients compared with aflibercept patients persisted with their index agent at month 12 (32.9\% vs $43.2 \%$, respectively). Secondly, a higher proportion of ranibizumab patients switched to aflibercept compared with the proportion of aflibercept patients who switched to ranibizumab at month $6(5.5 \%$ vs $1.5 \%)$ and month 12 (10.2\% vs $2.3 \%)$.

Finally, there was a continued gradual increase in the probability of discontinuation or switching among the two cohorts during a 12-month period (Fig. 5). However, there was a lower probability of discontinuation or switching for the aflibercept cohort relative to the ranibizumab cohort at month 6 (adjusted hazard ratio 0.86 ; 95\% CI 0.78-0.94) and month 12 (adjusted hazard ratio 0.76 ; 95\% CI 0.69-0.83) (Fig. 5). The divergence in the probability of discontinuation or switching occurred at 3060 days after index treatment. The Kaplan-Meier curves remained parallel for the two cohorts after 2 months (60 days) of therapy.

\section{DISCUSSION}

\section{Primary Study Objective and Outcome}

In the current study, the dispensing patterns of ranibizumab and aflibercept were analyzed and compared using data recorded and deposited by Australian pharmacies in the IMS ${ }^{\circledR}$ AUS LRx 09/2015 database. The primary objective of this study was to investigate the dispensing patterns of ranibizumab and aflibercept for the treatment of nAMD on the basis of the number of units dispensed of each anti-VEGF agent 6 and 12 months after the index date. Our analysis showed that the numbers of ranibizumab and aflibercept units dispensed were comparable, as the 95\% CI limits of the adjusted mean difference between cohorts did not exceed 1.00 unit 
Table 2 The number of ranibizumab and aflibercept units dispensed for the ranibizumab and aflibercept cohorts at month 6 and month 12

\begin{tabular}{|c|c|c|}
\hline & Ranibizumab cohort & Aflibercept cohort \\
\hline \multicolumn{3}{|l|}{ Month 6 (180 days) } \\
\hline No. of patients & 1235 & 959 \\
\hline No. of units dispensed & 6527 & 4875 \\
\hline Mean $^{\mathrm{a}}$ & $5.3(1.3)$ & $5.1(1.4)$ \\
\hline Range & $2.0-11.0$ & $2.0-12.0$ \\
\hline Median $^{\mathrm{b}}$ & $5.0(4.0-6.0)$ & $5(4.0-6.0)$ \\
\hline Adjusted mean difference $^{c}$ & & $0.20(0.09-0.32)$ \\
\hline \multicolumn{3}{|l|}{ Month 12 (360 days) } \\
\hline No. of patients & 578 & 483 \\
\hline No. of units dispensed & 5169 & 4301 \\
\hline Mean $^{a}$ & $8.9(2.2)$ & $8.9(2.3)$ \\
\hline Range & $4.0-18.0$ & $4.0-20.0$ \\
\hline Median ${ }^{\mathrm{b}}$ & $9.0(8.0-10.0)$ & $9.0(7.0-10.0)$ \\
\hline Adjusted mean difference ${ }^{c}$ & & $0.04(-0.23$ to 0.30$)$ \\
\hline
\end{tabular}

Mean differences were adjusted for baseline Medication-Based Disease Burden Index scores. Analyses were conducted with the final month 6 and month 12 study cohorts

${ }^{a}$ The standard deviation is given in parentheses

b The interquartile range is given in parentheses

c The $95 \%$ confidence interval is given in parentheses

at either time point. We therefore conclude that ranibizumab and aflibercept are dispensed similarly by Australian pharmacies in the first year of treatment.

The dispensing frequencies reported here for ranibizumab and aflibercept in the first year of treatment are similar to the injection frequencies reported for both agents in previous studies investigating the use of Australian PBS-listed therapies for nAMD. A report published by the Australian Drug Utilisation Sub Committee (DUSC) in 2015 showed that patients who started treatment between December 2012 and November 2013 with 12-month follow-up received a mean of 9.3 ranibizumab injections and 8.3 aflibercept injections [7]. Although the DUSC study reported a lower mean difference of 1.0 injection for aflibercept-treated patients compared with ranibizumab-treated patients, the identification period for this study began upon PBS-listing of aflibercept, and thus may not reflect more recently established patterns of nAMD treatment in clinical settings. In addition, Gillies et al. [14] showed the mean number of injections did not significantly differ between the ranibizumab cohort (8.1) and the aflibercept cohort (8.0) for treatment-naive nAMD eyes in patients who completed 12 months of antiVEGF therapy, while a recent analysis of data from Australian EMR databases yielded a mean number of 9.3 ranibizumab injections in T\&E-treated eyes in the first year of treatment [17]. Our findings show that the 12-month dispensing patterns for ranibizumab and aflibercept are consistent with real-world injection frequency data and support the use of 

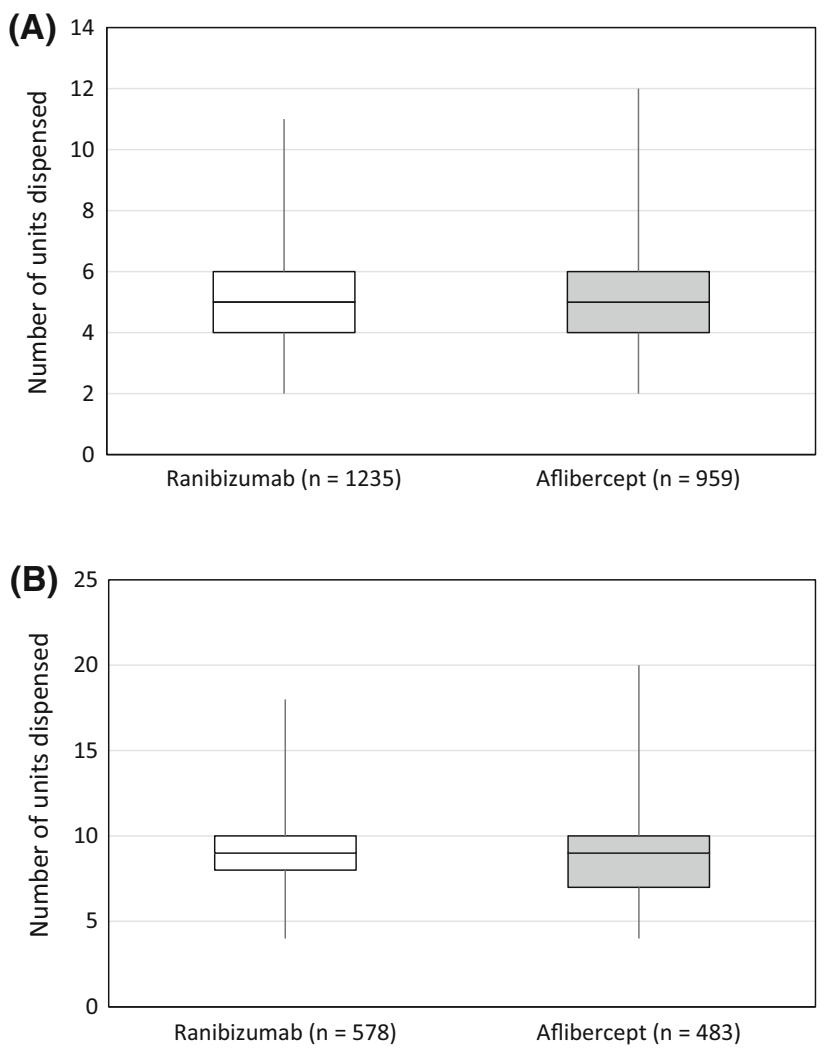

Fig. 2 The mean number of recorded anti-vascular endothelial growth factor units dispensed for the final persistent ranibizumab and aflibercept cohorts at month 6 (a) and month 12 (b)

dispensing data as a proxy for injection data when these are unavailable. Furthermore, the mean number of ranibizumab units dispensed during the first year of treatment was lower than the expected 13 ranibizumab doses under a monthly regimen as per the product label and further supports the adoption of the T\&E protocol in Australian clinics for the treatment of nAMD [9].

Of note, the mean numbers of dispensed units/injections of ranibizumab and aflibercept reported here and by others in the first year of nAMD treatment for Australian patients $[7,14,17]$ exceed those reported by studies that used US and European EMR databases. US nAMD patients were reported to receive a mean of 6.1-6.9 ranibizumab injections [16] and European patients were reported to receive a mean of 4.3-6.0 ranibizumab injections [30-32] in the first year of treatment. A recent analysis of US physician-level medical claims data also showed that in the first year of treatment the mean numbers of ranibizumab (4.9) and aflibercept (5.2) injections for nAMD patients were comparable despite differences in the labels between the two agents [15]. It is possible that "as needed" (pro re nata) treatment regimens may account, in part, for the lower anti-VEGF injection frequencies reported for Europe and the USA compared with Australia. However, despite differences in injection frequencies between geographical regions, the use of ranibizumab and aflibercept in the first year of treatment is comparable within countries, suggesting that the results of these studies may be generalized across populations.

\section{Secondary Study Outcomes}

No clinically meaningful differences were observed at month 6 or month 12 with regard to dispensing intervals between ranibizumab and aflibercept. Notably, the mean dispensing interval at month 12 for aflibercept (41.6 days) 


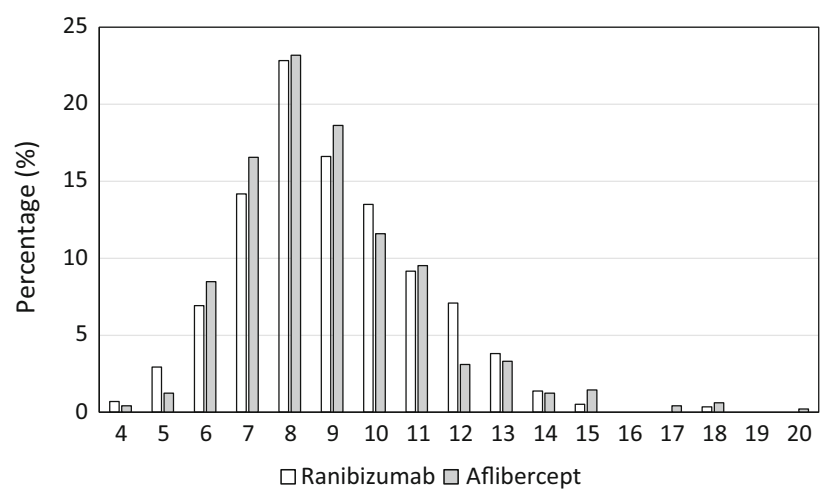

Fig. 3 Frequency distributions were constructed for the final month 12 cohort of persistent ranibizumab $(N=578)$ and aflibercept $(N=483)$ patients. These patients did not discontinue or switch treatment during this 12 -month post-index period. As the discontinuation gap was defined in this study as more than 120 days (i.e., 4 months), all patients included in this analysis had a record of receiving at least 4 units

Table 3 The dispensing interval for the ranibizumab and aflibercept cohorts at month 6 and month 12

\begin{tabular}{|c|c|c|}
\hline & Ranibizumab cohort & Aflibercept cohort \\
\hline \multicolumn{3}{|l|}{ Month 6 (180 days) } \\
\hline No. of patients & 1235 & 959 \\
\hline No. of dispensing intervals & 5292 & 3916 \\
\hline Mean interval (days) ${ }^{\mathrm{a}}$ & $35.3(19.2)$ & $36.8(20.0)$ \\
\hline Range (days) & $1.0-120.0$ & $1.0-120.0$ \\
\hline Median (days) ${ }^{\mathrm{b}}$ & $31.0(25.0-43.0)$ & $33.0(25.0-47.0)$ \\
\hline Adjusted mean difference (days) ${ }^{c}$ & & $-1.59(-2.51$ to -0.67$)$ \\
\hline \multicolumn{3}{|l|}{ Month 12 (360 days) } \\
\hline No. of patients & 578 & 483 \\
\hline No. of dispensing intervals & 4591 & 3818 \\
\hline Mean interval (days) ${ }^{a}$ & $41.2(20.9)$ & $41.6(20.4)$ \\
\hline Range (days) & $1.0-120.0$ & $1.0-120.0$ \\
\hline $\operatorname{Median}(\text { days })^{\mathrm{b}}$ & $37.0(28.0-54.0)$ & $39.0(28.0-55.0)$ \\
\hline Adjusted mean difference $(\text { days })^{c}$ & & $-0.40(-1.70$ to 0.91$)$ \\
\hline
\end{tabular}

Mean differences were adjusted for baseline Medication-Based Disease Burden Index scores. Analyses were conducted with the final month 6 and month 12 study cohorts.

a The standard deviation is given in parentheses

b The interquartile range is given in parentheses

c The 95\% confidence interval is given in parentheses

is consistent with the approximately 45 day mean injection interval expected under the recommended 8-week dosing schedule in the first year of treatment (i.e., 360 days/8 injections). In contrast, the mean dispensing interval for ranibizumab (41.2 days) is longer than 

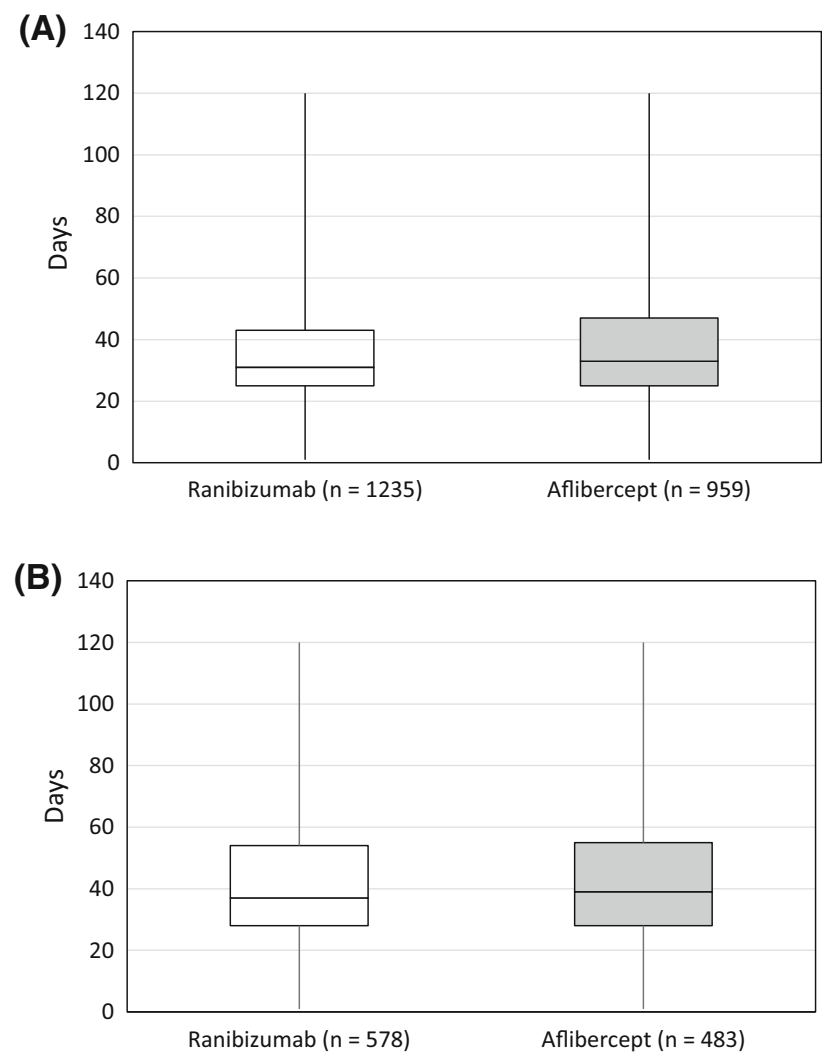

Fig. 4 Dispensing interval distributions for the final persistent ranibizumab and aflibercept cohorts at month 6 (a) and month $12(\mathbf{b})$

Table 4 The number and proportion of patients who persisted with, switched from, discontinued, or restarted index treatment at month 6 and month 12

\begin{tabular}{lllc}
\hline Cohort & Characteristics & Ranibizumab cohort & Aflibercept cohort \\
\hline Month 6 (180 days) & Total number of patients (primary analysis set) & $2412(100 \%)$ & $1711(100 \%)$ \\
& Persisted with index treatment & $1235(51.2 \%)$ & $959(56.0 \%)$ \\
& Switched to comparator treatment & $133(5.5 \%)$ & $25(1.5 \%)$ \\
& Discontinued index treatment & $969(40.2 \%)$ & $678(39.6 \%)$ \\
& Restarted index treatment & $75(3.1 \%)$ & $49(2.9 \%)$ \\
Month 12 (360 days) & Total number of patients (primary analysis set) & $1758(100 \%)$ & $1119(100 \%)$ \\
& Persisted with index treatment & $578(32.9 \%)$ & $483(43.2 \%)$ \\
& Switched to comparator treatment & $179(10.2 \%)$ & $26(2.3 \%)$ \\
& Discontinued index treatment & $855(48.6 \%)$ & $512(45.8 \%)$ \\
& Restarted index treatment & $146(8.3 \%)$ & $98(8.8 \%)$ \\
\hline
\end{tabular}

The number of patients refers to the patients in the primary analysis set 

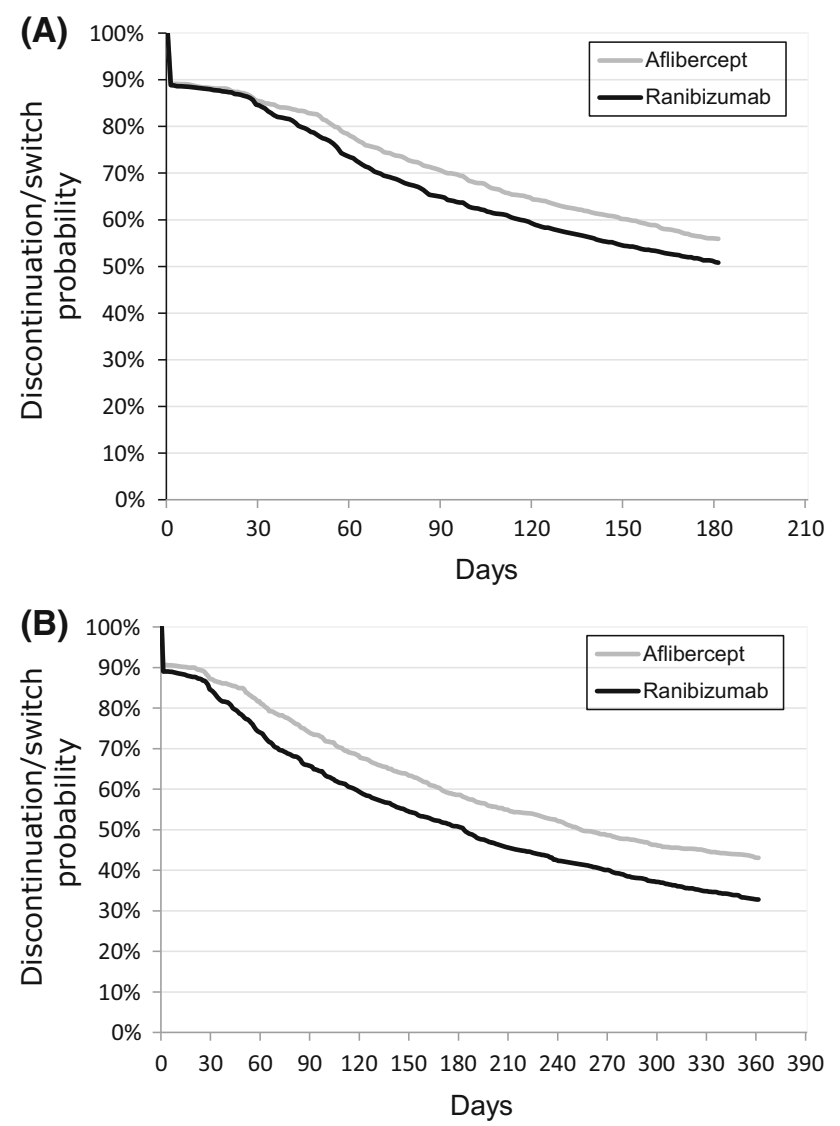

Fig. 5 Kaplan-Meier curves for time to discontinuation or switch of index anti-vascular endothelial growth factor treatment at month 6 (180 days; a) and month 12 (360 days; b)

the approximately 28 day mean interval expected under a monthly dosing regimen (i.e., 360 days/13 injections) in the first year of treatment. These results suggest that Australian ophthalmologists administer ranibizumab at intervals that differ from those recommended on the label, and give further credence to the common adoption of a T\&E regimen in Australia.

A lower proportion of ranibizumab patients than aflibercept patients persisted with the index treatment at month 12 , with the proportion of patients switching to the comparator agent higher for the ranibizumab cohort at both assessment time points. Aflibercept was first listed on the Australian PBS for the treatment of nAMD in December 2012, and it is possible that the availability of a new treatment option may have been appealing to clinicians after 2012, contributing to the higher level of switching to the comparator observed for the ranibizumab cohort compared with the aflibercept cohort $[7,25]$. This hypothesis is further supported by the pattern of the Kaplan-Meier curves illustrated in Fig. 5. There are two important observations from the Kaplan-Meier curves for the time to discontinuation or switch of the index anti-VEGF treatment: (1) the gradual continuous reduction in persistence with the index drug over time in both cohorts and (2) divergence between the cohorts occurred only at 30-60 days after the index treatment and remained parallel thereafter. It is important to note that the data used in the current study were collected from regional pharmacies across Australia and that some patients may have: (1) switched from their original pharmacy during the identification period to a pharmacy that is 
not registered with the IMS ${ }^{\circledR}$ AUS LRx 09/2015 database; (2) switched to unlicensed bevacizumab for the treatment of nAMD during the identification period; or (3) switched between two or more pharmacies that are in the same geographical region during the identification period. Such patients may have been recorded in the IMS ${ }^{\circledR}$ AUS LRx 09/2015 database as having switched from or discontinued use of their index agent, thereby contributing to the appreciable proportion of patients who discontinued their index therapy ( $48.6 \%$ for ranibizumab and $45.8 \%$ for aflibercept) at month 12 . This scenario may also account, in part, for the differences in the proportion of ranibizumab and aflibercept patients who switched to the comparator agent at both assessment time points together with the lower risk of discontinuation or switching for aflibercept relative to ranibizumab. The divergence in Kaplan-Meier curves between the two cohorts between 30 and 60 days after the index treatment and not beyond suggests that prescribing preference rather than a difference in treatment efficacy is the predominant driver of drug use discontinuation or switching. It should also be considered that statistically significant differences reported in this study are a consequence of the large number of patients included in the analysis and do not necessarily reflect meaningful differences in routine clinical settings.

\section{Study Limitations}

Although no difference in the dispensing patterns of ranibizumab and aflibercept in the first year of treatment was concluded from our analysis, it is important to note the limitations of this study. First, the data used in this analysis were collected for healthcare transaction purposes rather than for research purposes, and it was assumed that patients with a recorded receipt for a given anti-VEGF agent were prescribed that agent for the treatment of nAMD. During the identification period, aflibercept was licensed for the treatment of nAMD only, while ranibizumab was licensed for several indications (i.e., nAMD, diabetic macular edema, and retinal vein occlusion) $[7,9,10,26,27]$. The inclusion of ranibizumab prescriptions for indications other than nAMD would conceivably result in an overestimation of the number of ranibizumab units dispensed in the final study cohort, thereby artificially increasing the mean number of ranibizumab units dispensed and lowering the mean dispensing interval. However, we believe that the impact of ranibizumab prescriptions for indications other than nAMD on the final dataset used here is likely to be negligible as the cost of ranibizumab was reimbursed only for the treatment of nAMD by the PBS during the identification period $[9,26]$. Second, baseline patient characteristics such as age and sex were not well populated in the IMS ${ }^{\circledR}$ AUS LRx 09/2015 database and could not be used as covariates in the analyses presented here. Although baseline patient comorbidities (based on MBDBI scores) were comparable between the two cohorts, differences in baseline patient characteristics may influence the findings reported here. Third, information regarding the injection of ranibizumab or aflibercept (including the date of injection or the number of injections administered) is not captured in the database; hence, the number of units dispensed and the date of dispensation were used as a proxy for these data. Although our analysis supports the value of dispensing data as a proxy for injection data in Australia, our results may not reflect anti-VEGF use in other countries. Fourth, although the frequency of dispensing is suggestive of bilateral treatment, laterality is not consistently captured in the database and was not controlled for in this study. Fifth, regional differences in anti-VEGF treatment rates across Australia were not evaluated here despite treatment rates being higher for more densely populated states or territories (e.g., Australian Capital Territory, New South Wales, and Tasmania) than for less densely populated regions (e.g., Northern Territory) [7]. In addition, only sites using the IMS ${ }^{\circledR}$ AUS LRx 09/2015 database were included in this study, which may result in a selection bias. This database has $31 \%$ coverage of all retail pharmacies; therefore, the generalizability of the study results may extend only to persons claiming prescribed medication from these pharmacies in Australia. Finally, the IMS $^{\circledR}$ AUS LRx 09/2015 database 
does not contain information on outcomes of clinical interest, such as visual acuity or retinal anatomical features (e.g., retinal fluid, retinal atrophy, pigment epithelial detachment, and hemorrhage); thus, further data sources are required to explore the relationship between injection frequency and visual outcomes in routine clinical settings. It is important to note that all limitations of the IMS $^{\circledR}$ AUS LRx $09 / 2015$ database apply equally to both final study cohorts, and the results or interpretations of this study are not expected to be systematically biased in favor of either anti-VEGF agent.

\section{CONCLUSION}

Pharmacy claims databases offer a rich source of information to analyze the treatment patterns of anti-VEGF agents used for the treatment of nAMD in real-world settings. This study demonstrated that for patients included in the Australian IMS ${ }^{\circledR}$ AUS LRx 09/2015 database who met the study selection criteria, there was no difference in the dispensing patterns of ranibizumab and aflibercept 6 and 12 months after treatment initiation. The number of ranibizumab and aflibercept units dispensed was also comparable to the number of injections administered to treatment-naive nAMD patients in the first year of treatment reported by other studies and supports the use of dispensing data as a proxy for injection data. Our results also support the common use of a T\&E protocol in Australia. Further studies exploring longer-term dispensing patterns (e.g., 2 years or more after the initiation of treatment) would provide additional insight into the real-world use of anti-VEGF agents and how this may impact national healthcare schemes for the treatment of nAMD.

\section{ACKNOWLEDGEMENTS}

Sponsorship for this study, article processing charges, and the open access fee were funded by Novartis Pharma AG. All authors take complete responsibility for the integrity of the data and accuracy of the data analysis. All named authors meet the International Committee of Medical
Journal Editors criteria for authorship for this article, take responsibility for the integrity of the work as a whole, and have given final approval for the version to be published. The authors received medical writing support for this manuscript from David A. Magee (Novartis Ireland Ltd, Dublin, Ireland).

Disclosures. Adrian Skelly is an employee of Novartis Pharma AG, Basel, Switzerland. Vladimir Bezlyak is an employee of Novartis Pharma AG, Basel, Switzerland. Hans-Joachim Carius is an employee of QuintilesIMS Frankfurt, Germany, funded by Novartis to perform the statistical analyses for this study and has no conflict of interest. Fred Chen receives research funding from Lions Eye Institute and the University of Western Australia. He receives research funding from Bayer and Novartis to conduct investigator-initiated trials but does not receive consulting fees from either company.

Compliance with ethics guidelines. This study was designed, conducted, and reported in accordance with the guidelines for good pharmacoepidemiology practices of the International Society for Pharmacoepidemiology, the Strengthening the Reporting of Observational Studies in Epidemiology guidelines, and the ethical principles stated in the Declaration of Helsinki. For this study, all patient identifiers were removed; thus, formal patient informed consent was not required.

Data availability. The datasets generated and/or analyzed during the current study are not publicly available as they were obtained from a proprietary database through a licensing agreement.

Open Access. This article is distributed under the terms of the Creative Commons Attribution-NonCommercial 4.0 International License (http://creativecommons.org/licenses/ by-nc/4.0/), which permits any noncommercial use, distribution, and reproduction in any medium, provided you give appropriate credit to the original author(s) and the source, provide a link to the Creative Commons license, and indicate if changes were made. 


\section{REFERENCES}

1. Congdon N, O'Colmain B, Klaver CC, Klein R, Munoz B, Friedman DS, et al. Causes and prevalence of visual impairment among adults in the United States. Arch Ophthalmol. 2004;122(4):477-85.

2. Resnikoff S, Pascolini D, Etya'ale D, Kocur I, Pararajasegaram R, Pokharel GP, et al. Global data on visual impairment in the year 2002. Bull World Health Organ. 2004;82(11):844-51.

3. Deloitte Access Economics. Eyes on the future: a clear outlook on age-related macular degeneration. Report prepared for the Macular Degeneration Foundation. October 2011. https://www. mdfoundation.com.au/sites/default/files/Deloitte_ Eyes_on_the_Future_Report_web_1_0.pdf. Accessed Aug 2017.

4. Galloway NR, Amoaku WMK, Galloway PH, Browning AC. The ageing eye. In: Common eye diseases and their management, London: Springer; 2006.

5. Ambati J, Fowler BJ. Mechanisms of age-related macular degeneration. Neuron. 2012;75(1):26-39.

6. Kovach JL, Schwartz SG, Flynn HW Jr, Scott IU. Anti-VEGF treatment strategies for wet AMD. J Ophthalmol. 2012;2012:786870.

7. Drug Utilisation Sub-committee. In: Pharmaceutical Benefits Scheme, editor. Age related macular degeneration: utilisation analysis. Canberra, Australia; 2015.

8. Spaide R. Ranibizumab according to need: a treatment for age-related macular degeneration. Am J Ophthalmol. 2007;143(4):679-80.

9. Australian Government, Department of Health, Therapeutic Goods Administration. Lucentis ${ }^{\circledR}$. Ranibizumab. Consumer medicine information. 2017. https://www.ebs.tga.gov.au/ebs/picmi/ picmirepository.nsf/pdf?OpenAgent\&id=CP-2010CMI-07299-3. Accessed Aug 2017.

10. Australian Government, Department of Health, Therapeutic Goods Administration. EYLEA ${ }^{\circledR}$. Aflibercept. Consumer medicine information. 2016. https://www.ebs.tga.gov.au/ebs/picmi/ picmirepository.nsf/pdf?OpenAgent\&id=CP-2012CMI-02075-3. Accessed Aug 2017.

11. Gupta OP, Shienbaum G, Patel AH, Fecarotta C, Kaiser RS, Regillo CD. A treat and extend regimen using ranibizumab for neovascular age-related macular degeneration clinical and economic impact. Ophthalmology. 2010;117(11):2134-40.
12. Heier JS, Brown DM, Chong V, Korobelnik JF, Kaiser PK, Nguyen QD, et al. Intravitreal aflibercept (VEGF Trap-Eye) in wet age-related macular degeneration. Ophthalmology. 2012;119(12):2537-48.

13. Schmidt-Erfurth U, Kaiser PK, Korobelnik JF, Brown DM, Chong V, Nguyen QD, et al. Intravitreal aflibercept injection for neovascular age-related macular degeneration: ninety-six-week results of the VIEW studies. Ophthalmology. 2014;121(1): 193-201.

14. Gillies MC, Nguyen V, Daien V, Arnold JJ, Morlet N, Barthelmes D. Twelve-month outcomes of ranibizumab vs. aflibercept for neovascular age-related macular degeneration: data from an observational study. Ophthalmology. 2016;123(12):2545-53.

15. Ferreira A, Sagkriotis A, Olson M, Lu J, Makin C, Milnes F. Treatment frequency and dosing interval of ranibizumab and aflibercept for neovascular age-related macular degeneration in routine clinical practice in the USA. PLoS One. 2015;10(7): e0133968.

16. Holekamp NM, Liu Y, Yeh WS, Chia Y, Kiss S, Almony A, et al. Clinical utilization of anti-VEGF agents and disease monitoring in neovascular age-related macular degeneration. Am J Ophthalmol. 2014;157(4):825-33.

17. Johnston RL, Carius HJ, Skelly A, Ferreira A, Milnes F, Mitchell P. A retrospective study of ranibizumab treatment regimens for neovascular age-related macular degeneration (nAMD) in Australia and the United Kingdom. Adv Ther. 2017;34(3):703-12.

18. Kiss S, Dugel PU, Wilson K, Huang A, Smith D, Varker $\mathrm{H}$, et al. Treatment patterns and associated costs of anti-VEGF therapy for neovascular age-related macular degeneration. Invest Ophthalmol Vis Sci. 2014;55(13):5596.

19. Lotery AJ, Regnier S. Patterns of ranibizumab and aflibercept treatment of central retinal vein occlusion in routine clinical practice in the USA. Eye (Lond). 2015;29(3):380-7.

20. Souied EH, Dugel PU, Ferreira A, Hashmonay R, Lu J, Kelly SP. Severe ocular inflammation following ranibizumab or aflibercept injections for age-related macular degeneration: a retrospective claims database analysis. Ophthalmic Epidemiol. 2016;23(2):71-9.

21. Andrews EB, Arellano FM, Avorn J, Bortnichak EA, Chen R, Dai WJS, et al. Guidelines for good pharmacoepidemiology practice (GPP). Pharmacoepidemiol Drug Saf. 2016;25(1):2-10.

22. Vandenbroucke JP, von Elm E, Altman DG, Gotzsche PC, Mulrow CD, Pocock SJ, et al. 
Strengthening the reporting of observational sudies in eidemiology (STROBE): explanation and elaboration. Int J Surg. 2014;12(12):1500-24.

23. World Medical Association. World Medical Association Declaration of Helsinki: ethical principles for medical research involving human subjects. JAMA. 2013;310(20):2191-4.

24. Australian Government, Department of Health. The Pharmaceutical Benefits Scheme-aflibercept. http://www.pbs.gov.au/medicine/item/10505X2168 D (2017). Accessed Jun 2017.

25. Australian Government, Department of Health. The Pharmaceutical Benefits Scheme-ranibizumab. http://www.pbs.gov.au/medicine/item/10138N10374B (2017). Accessed Jun 2017.

26. mivision. Lucentis Listed on PBS for DME and RVO. https://www.mivision.com.au/lucentis-listed-onpbs-for-dme-and-rvo/ (2015). Accessed Aug 2017.

27. mivision. Eylea approved for diabetic macular edema. https://www.mivision.com.au/eylea-approved-fordiabetic-macular-edema/ (2015). Accessed Aug 2017.

28. George J, Vuong T, Bailey MJ, Kong DC, Marriott JL, Stewart K. Development and validation of the medication-based disease burden index. Ann Pharmacother. 2006;40(4):645-50.

29. Thomas M, Mousa SS, Mousa SA. Comparative effectiveness of aflibercept for the treatment of patients with neovascular age-related macular degeneration. Clin Ophthalmol. 2013;7:495-501.

30. Holz FG, Bandello F, Gillies M, Mitchell P, Osborne A, Sheidow T, et al. Safety of ranibizumab in routine clinical practice: 1-year retrospective pooled analysis of four European neovascular AMD registries within the LUMINOUS programme. Br J Ophthalmol. 2013;97(9):1161-7.

31. Ross AH, Donachie PH, Sallam A, Stratton IM, Mohamed Q, Scanlon PH, et al. Which visual acuity measurements define high-quality care for patients with neovascular age-related macular degeneration treated with ranibizumab? Eye. 2013;27(1):56-64.

32. Writing Committee for the UK Age-Related Macular Degeneration EMR Users Group. The neovascular age-related macular degeneration database: multicenter study of 92976 ranibizumab injections: report 1: visual acuity. Ophthalmology. 2014;121(5): 1092-101. 